\title{
Tendências e Parâmetros Genéticos para Características Pré-desmama em Bovinos da Raça Charolês Criados no Rio Grande do Sul
}

\author{
Henrique Dorneles Fernandes ${ }^{1}$, Gilka Berenice Barbosa Ferreira², Paulo Roberto Nogara Rorato²
}

RESUMO - O objetivo deste trabalho foi a determinação, por intermédio do cálculo da tendência genética, do progresso genético de rebanhos Charolês criados no Estado do Rio Grande do Sul, para que os resultados sirvam como base para a elaboração de futuros programas de melhoramento genético para esta raça.Os registros de peso foram coletados pelo PROMEBO (Programa de Melhoramento Bovino). As análises foram realizadas no Laboratório de Melhoramento Animal do Departamento de Zootecnia da Universidade Federal de Santa Maria. Foram estimadas herdabilidades $\left(\mathrm{h}^{2}\right)$ e as DEP's (diferença esperada na progênie), por intermédio do programa computacional DFREML (Boldman et al., 1995), para determinar as tendências genéticas anuais, direta e materna, das características peso ao nascer (PN) e ganho de peso do nascimento à desmama (GPND), com o auxílio do procedimento PROC REG (SAS, 1996). Foram encontradas $\mathrm{h}^{2}$ direta e materna de 0,54 e 0,12 para PN e 0,12 e 0,05 para GPND, respectivamente. Verificou-se tendência genética direta, não-significativa de -10,46 e 40,85 g/ano, além de tendência genética materna de 2,38 e -10,34 g/ano, para PN e GPND, respectivamente. Estes resultados indicam que esta população se encontra sem progresso genético, embora tenha sido verificado progresso fenotípico significativo para ambas as características. De posse destas informações, torna-se evidente a urgência da adoção de um programa de melhoramento genético para este rebanho, considerando que, em uma população que está progredindo fenotipicamente, a resposta ao programa adotado seria mais rápida.

Palavras-chave: ganho de peso pré-desmama, peso ao nascer, progresso genético

\section{Trends and Genetic Parameters of Growth Trait of Charolais Herd Raised in the Rio Grande do Sul State}

\begin{abstract}
The objective of this work was to determine, through the genetic trend, the genetic progress of Charolais herds raised in the Rio Grande do Sul State, and the results utilized in programs of genetic improvement for this breed. The data were collected by PROMEBO (Beef Breeding Program) and the analyses were performed at the Animal Breeding Laboratory of the Animal Science Department of the Universidade Federal de Santa Maria. It was estimated the heritabilities $\left(\mathrm{h}^{2}\right)$ and the breeding values (BV) by means of the DFREML program (Boldman et al., 1995) to determine the annual direct and maternal genetic tendency for birth weight (BW) and weight gain from birth to weaning (WGBW) with the PROC REG (SAS, 1996). The results showed direct and maternal heritabilities of .54 and .12 for BW and .12 and .05 for WGBW, respectively. There were no significant direct trend of -10.46 and $40.85 \mathrm{~g} / \mathrm{year}$ and a maternal genetic trend of 2.38 and $-10.34 \mathrm{~g} /$ year, for BW and WGBW, respectively. These results indicate that there was no genetic progress in this population, although significant phenotypic progresses for both characteristics were verified. This results leads to conclusion that there is a necessity of adoption of a genetic improvement program in this breed, considering that the population is improving phenotipically and the results would show in a short period of time.
\end{abstract}

Key Words: birth weight, genetic progress, weight gain from birth to weaning

\section{Introdução}

Os crescentes esforços, nos últimos tempos, no sentido de transformar a exploração da pecuária de corte em uma atividade mais eficiente e rentável, transformaram o setor em diferentes proporções criando basicamente dois grupos bastante distintos. Por um lado, os criadores descapitalizados, com sistemas de criação mais extensivos, lutam para tentar manter-se no mercado, enquanto os mais tecnificados buscam sempre novas alternativas para intensificar a sua produção e ampliar o seu retorno financeiro. A genética dos rebanhos é uma das principais preocupações atuais do produtor que busca novas alternativas, pois é sabido que a eficiência na resposta a determinado manejo nutricional é diretamente proporcional à superioridade genética dos animais utilizados na propriedade.

A busca por animais mais pesados na fase de acabamento faz com que muitos produtores selecio-

\footnotetext{
${ }^{1}$ Zootecnista, Mestre, Universidade Federal de Santa Maria. Correspondências: Rua Mariz e Barros, 391 CEP: 97542-450, Alegrete, RS. E.mail: hdorneles@gpsnet.com.br

2 Doutores, Professores Adjuntos do Departamento de Zootecnia - UFSM. E.mail: gbbf@ccr.ufsm.br; rorato@ccr.ufsm.br
} 
nem os reprodutores considerando apenas este critério, esquecendo que este fato poderá resultar em problemas de distocia, principalmente para a raça Charolês, devido ao elevado peso ao nascer dos animais desta raça.

É de suma importância a realização de melhoramento genético em uma população, no decorrer dos anos, paralelamente à evolução fenotípica, pois o progresso fenotípico de uma população tende a alcançar um limite, e isso ocorrerá quando o animal estiver respondendo dentro de sua capacidade genética máxima, às melhorias ambientais adotadas. A partir deste ponto, se não houver progresso genético, não ocorrerá progresso fenotípico.

A implantação de um programa de melhoramento genético dos animais é fundamental para alcançar melhorias na genética de um rebanho. Porém, depois de implantado, é necessário que seja feita, periodicamente, a verificação de sua eficiência (Mello, 1999), ou seja, verificar se está ocorrendo o progresso genético esperado, para que, caso contrário, sejam tomadas as providências necessárias para que isso aconteça.

Uma forma de promover este acompanhamento é avaliar o progresso genético alcançado pelo rebanho ao longo do tempo, ou avaliar a variação do ganho genético anual dos animais. Uma das maneiras de se avaliar esse progresso é por intermédio do cálculo da tendência genética da população, que se torna possível quantificando quanto da variação anual do rebanho tem origem genética, fazendo-se necessário que a mudança fenotípica seja decomposta nos seus componentes genéticos e de ambiente (Oliveira et al., 1995). Isto se consegue por intermédio do cálculo das DEP's (diferença esperada na progênie) dos animais da população.

Também torna-se de grande importância, quando se pretende uma seleção genética eficiente, a estimativa da herdabilidade direta e materna da característica estudada na população, que é uma das principais ferramentas do melhoramento animal usado pelos criadores e melhoristas (Biffani et al., 1999). Isto porque características com estimativas de baixa herdabilidade não apresentam respostas satisfatórias ao trabalho de seleção dos animais.

Separar a fração genética da ambiental e determinar a importância relativa dos efeitos aditivos genéticos direto e materno se torna necessário quando se pretende formular planos de melhoramento animal (Ferreira et al., 1999), principalmente na fase pré-desmama, quando esses efeitos têm grande influência sobre a resposta do animal.

R. Bras. Zootec., v.31, n.1, p.321-330, 2002 (suplemento)
Em vista disso, e também pelo fato de que existem poucos trabalhos seguindo esta linha de pesquisa no sul do País, especialmente em relação às raças européias, o que indica um menor acompanhamento da genética dessas populações, é que os objetivos deste trabalho foram: analisar a capacidade genética, através do cálculo da tendência genética aditiva direta, e materna, das características peso ao nascer e ganho de peso do nascimento à desmama de animais de rebanhos da raça Charolês criados no Estado do Rio Grande do Sul para que estes resultados sirvam como base para a elaboração de futuros programas de melhoramento genético específicos para esta raça; analisar o progresso fenotípico da população para que seja verificada a influência da capacidade genética no desempenho dos animais; calcular as estimativas de herdabilidades direta e materna da população; e identificar a presença de animais melhoradores, conforme o critério de seleção preestabelecido.

\section{Material e Métodos}

Os dados, utilizados neste trabalho, referentes às características peso ao nascer e ganho de peso do nascimento à desmama (205 dias) de rebanhos da raça Charolês, foram coletados em todo o Estado do Rio Grande do Sul entre os anos de 1983 e 1998 pelo PROMEBO (Programa de Melhoramento de Bovinos) com sede em Pelotas, RS. Os arquivos utilizados para as análises foram editados e analisados no Laboratório de Melhoramento Animal do Departamento de Zootecnia da Universidade Federal de Santa Maria.

Segundo Mota et al. (1994), o clima desta região caracteriza-se por depender do movimento de enormes massas de ar de origem oceânica, tropical e polar. A temperatura média anual do Estado fica em torno de $18^{\circ} \mathrm{C}$, variando de 13,7 a $23,6^{\circ} \mathrm{C}$, médias anuais mínimas e máxima, registrados num período de 30 anos. A precipitação pluviométrica total média anual fica em torno de $1.620 \mathrm{~mm}$, com chuvas meteorologicamente bem distribuídas durante o ano, mas, sob o ponto de vista climatológico, mal distribuídas, devido à enorme diferença de evaporação do inverno para o verão, não satisfazendo as necessidades mínimas de algumas culturas. No verão, são registradas temperaturas elevadas (superiores a $42^{\circ} \mathrm{C}$ em algumas cidades) e reduções das chuvas em fevereiro. Do outono até a primavera, há grande 
possibilidade de ocorrência de geadas, podendo prejudicar a agropecuária. Embora compreendido na Zona Temperada da classificação de Köeppen, o Rio Grande do Sul possui um clima caracteristicamente subtropical, devido à influência marítima.

Os animais foram distribuídos em grupos contemporâneos do nascimento à desmama de acordo com o sexo, a estação de nascimento, o criador, o rebanho de origem, o código alimentar, e a data Juliana de pesagem de cada indivíduo, sendo que animais sob influência dos mesmos efeitos foram colocados em grupos distintos, e estes enumerados de 1 a 557.

A editoração dos arquivos para análise foi realizada com o auxílio do programa estatístico SAS (Sas, 1996). Posteriormente, o programa computacional MTDFREML (Boldman et al., 1995) foi utilizado para estimar as DEP's (diferenças esperadas na progênie), para cada indivíduo desta população. Foram ainda estimados os parâmetros genéticos de herdabilidade direta e materna. O critério de convergência considerado para as análises com o DFREML foi de 10-6, sendo que a cada convergência o programa era reinicializado usando valores obtidos na análise anterior.

Os valores das DEP's individuais foram, então, utilizados para cálculo da variação ocorrida na freqüência dos genes no decorrer dos anos em que foram coletadas as informações.

Os animais foram separados de acordo com o ano de nascimento, para que pudessem ser obtidas as DEP's médias anuais para o período compreendido entre os anos de 1984 a 1998, para PN (peso ao nascer) e GPND (ganho de peso do nascimento à desmama), sendo que o conjunto de dados referente ao primeiro ano (1984) na verdade reuniu os animais nascidos nos anos de 1983 e 1984, devido ao fato de que no ano de 1983 o número de coletas foi extremamente pequeno (58 para PN e GPND) e, por isso, optou-se por analisá-los juntamente com o ano seguinte.

De posse das DEP's médias anuais, calculou-se a regressão das mesmas em relação ao ano de nascimento dos animais, utilizando o procedimento PROC REG do SAS (1996), seguindo um modelo matemático do tipo:

$$
\mathrm{Y}_{\mathrm{i}}=\mathrm{b}_{0}+\mathrm{b}_{1} \mathrm{X}_{\mathrm{i}}
$$

em que: $Y_{i}$ é a DEP média do iésimo ano de nascimento; $\mathrm{X}_{\mathrm{i}}$, o iésimo ano de nascimento; $\mathrm{b}_{0}$, a constante da equação; e $b_{1}$, o coeficiente de regressão linear.

Com isso tornou-se possível estimar, por meio da confecção da reta de tendência linear dos valores coletados, a tendência genética direta desta população.

Além desta tendência direta, foi calculada a tendência genética materna da população, usando para isso as DEP's maternas dos animais, obtidas nas mesmas análises realizadas para a determinação das DEP's diretas. É de suma importância a determinação da influência do efeito materno na população, segundo Hohenboken (1985), citado por Fries \& Albuquerque (1996), efeito materno é qualquer contribuição, influência ou impacto sobre o fenótipo de um indivíduo atribuível diretamente ao fenótipo de sua mãe.

Para efeito de comparação, também foi calculada a regressão dos valores médios anuais coletados, das características citadas, seguindo o mesmo padrão de análise das DEP's, por intermédio de modelos do mesmo tipo, em que: Yi é o peso ao nascer médio ou o ganho de peso pré-desmama médio do iésimo ano de nascimento, para que fossem obtidas as tendências fenotípicas da população, ou seja, para ser verificada a evolução total não apenas da genética do rebanho, mas também da porção não-genética deste.

A característica peso ao nascer foi analisada univariadamente, para cálculo das DEP's e dos parâmetros genéticos, por intermédio de um modelo animal com inclusão do efeito genético aditivo direto do animal, efeito genético materno, e os efeitos fixos de grupo contemporâneo do nascimento à desmama e de ano de nascimento, descrito da seguinte forma em termos matriciais:

$$
\mathrm{Y}=\mathrm{Z}_{\mathrm{a}}+\mathrm{Z}_{\mathrm{b}}+\mathrm{W}_{\mathrm{g}}+\mathrm{W}_{\mathrm{m}}+\mathrm{e}
$$

em que: $Y=$ vetor de observação; $Z_{a}=$ matriz de incidência do efeito fixo de grupo contemporâneo do nascimento à desmama; $\mathrm{Z}_{\mathrm{b}}=$ matriz de incidência do efeito fixo de ano de nascimento; $\mathrm{W}_{\mathrm{g}}=$ matriz de incidência dos efeitos genéticos diretos; $\mathrm{W}_{\mathrm{m}}=$ matriz de incidência dos efeitos genéticos maternos; $\mathrm{e}=$ erro aleatório residual.

Segundo Fernandes \& Ferreira (2000), para características pré-desmama, a utilização de um modelo animal com a inclusão dos efeitos direto e materno não apresentou diferença significativa quanto às estimativas de coeficientes de (co) variância e DEP, em relação ao modelo que incluía também o efeito de ambiente permanente. Scarpati \& Lôbo (1999) concluíram que as estimativas de componentes de (co) variância para peso ao nascer podem ser feitas utilizando um modelo que não inclua os efeitos de ambiente permanente e genético materno simultane- 
amente, pois estes se ajustam tão bem aos dados quanto um modelo mais completo.

Foram analisadas as informações de 6.529 animais, distribuídos por ano de nascimento, conforme mostrado na Figura 1.

$\mathrm{O}$ ganho de peso do nascimento à desmama foi analisado, também univariadamente, para o cálculo das DEP's e dos parâmetros genéticos, por intermédio de um modelo animal com inclusão do efeito aditivo direto do animal, efeito não correlacionado de ambiente permanente, efeito genético materno, e efeitos fixos de grupo contemporâneo do nascimento à desmama e ano de nascimento, mostrado em termos matriciais da seguinte forma:

$$
\mathrm{Y}=\mathrm{Z}_{\mathrm{a}}+\mathrm{Z}_{\mathrm{b}}+\mathrm{W}_{\mathrm{g}}+\mathrm{W}_{\mathrm{m}}+\mathrm{W}_{\mathrm{p}}+\mathrm{e}
$$

em que: $Y, Z_{a}, Z_{b}, W_{g}, W_{m}$, e = como descritos anteriormente; $\mathrm{Wp}=$ matriz de incidência dos efeitos de ambiente permanente.

Nesta análise, foram utilizadas as informações de 9.808 animais. A distribuição dos animais por ano de nascimento é mostrada na Figura 2.

Foi realizado o cálculo da correlação das DEP's diretas das características peso ao nascer e ganho de peso do nascimento à desmama, sendo que, devido ao fato de a característica de ganho apresentar número maior de dados, foram feitas eliminações dos animais que apresentavam registro de ganho de peso e não de peso ao nascer, sendo que todos os animais presentes nos registros de peso ao nascer também apareciam nos do ganho de peso pré-desmama. O cálculo do ganho de peso dos animais que não apresentavam

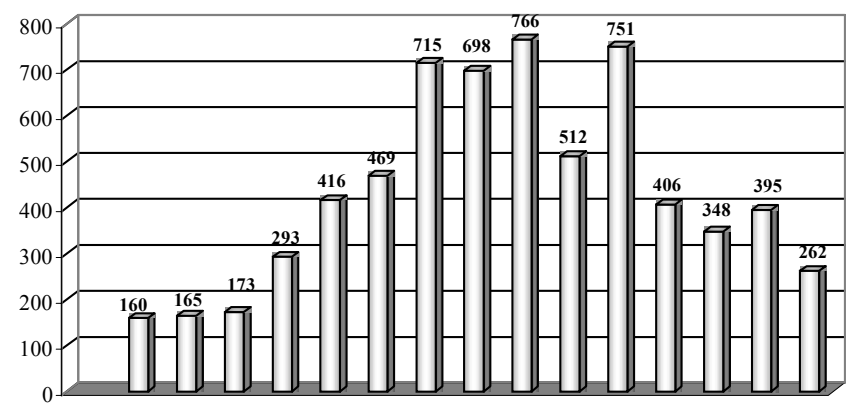

Figura 1 - Número de animais por ano de nascimento, de 1984 a 1998, para a característica peso ao nascer.

Figure 1 - Number of animals by year from birth, from 1984 to 1998 for the birth weight.

R. Bras. Zootec., v.31, n.1, p.321-330, 2002 (suplemento)

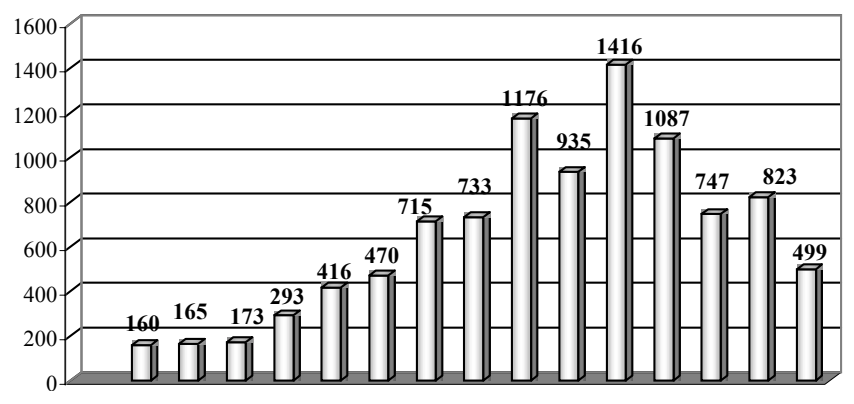

Figura 2 - Número de animais por ano de nascimento, de 1984 a 1998, para a característica ganho de peso do nascer à desmama.

Figure 2 - Number of animals by birth year, from 1984 to 1998, for the weight gain from birth to weaning.

registro de peso ao nascer foi feito utilizando-se o valor médio desta característica para esta raça. Após estas eliminações, correlacionou-se através do procedimento PROC CORR (SAS, 1996) as DEP's diretas das duas características de 6.529 animais.

Devido à crescente exigência dos produtores desta raça por animais que produzam progênies com menor peso ao nascimento, devido a problemas de distocia, porém sem redução no peso à desmama, o que se conseguiria com animais com DEP's negativas para o peso ao nascer e positivas para o ganho de peso do nascer à desmama; foi realizado a ordenação das DEP's para estas duas características, por intermédio do procedimento PROC SORT (Sas, 1996) ou seja, dispor lado a lado as DEP's dos animais para que pudessem ser identificados os que conseguiam satisfazer as exigências de DEP's de peso ao nascer negativas e de ganho de peso do nascer à desmama positivas.

\section{Resultados e Discussão}

A população estudada apresentou pesos ao nascer variando de 21 a $66 \mathrm{~kg}$, com média de $41,55 \mathrm{~kg}$, valor este bastante alto, mesmo para uma raça de grande porte como a Charolês. Em relação a característica ganho de peso do nascimento à desmama, esta população apresentou valores médios de $154,79 \mathrm{~kg}$, com variação de 41,01 a $398,78 \mathrm{~kg}$.

Ferreira et al. (1999) encontraram, para animais da raça Hereford, peso ao nascer médio de $35,9 \mathrm{~kg}$ e ganho de peso pré-desmama de 166,6 kg; Mello (1999) encontrou valores semelhantes para animais 
Canchim, com peso ao nascer médio de $35,8 \mathrm{~kg}$ e ganho pré-desmama de 159,34 kg, já Mascioli et al. (1997) encontraram, para animais Canchim, peso ao nascer de 35,3 kg e ganhos pré-desmama de $173,6 \mathrm{~kg}$. $\mathrm{O}$ valor do ganho de peso do nascimento à desmama, encontrado neste trabalho, foi mais alto apenas quando comparado aos obtidos para raças zebuínas, como o Gir, cujos animais apresentaram ganhos médios de 103,32 kg (Euclides Filho et al., 2000), e o Tabapuã, com ganhos de peso médios de 135,71 kg (Silva et al., 1997a). Marques et al. (1999), trabalhando igualmente com animais de grande porte, como o Simental, encontraram alto peso médio ao nascer $(40,1 \mathrm{~kg})$ porém também foram altos os valores de ganho prédesmama $(178,7 \mathrm{~kg})$.

Esses resultados sugerem que, no período estudado, não deve ter ocorrido seleção para a característica de ganho de peso, e sim, provavelmente, para as características de peso à desmama e ao sobreano. A alta e positiva correlação genética entre estas características e a de peso ao nascimento fez com que esta também fosse aumentada.

O coeficiente de herdabilidade $\left(\mathrm{h}^{2}\right)$ direta estimada neste estudo (Tabela 1) para o peso ao nascimento $(0,54)$ mostrou-se mais alto que a maioria dos resultados verificados na literatura, o que indicaria uma resposta bastante eficiente desta característica à seleção, nesta população. A $\mathrm{h}^{2}$ materna, porém, mostrou-se mais baixa $(0,12)$, o que concorda com a

Tabela 1 - Estimativas de componentes de variância e coeficiente de herdabilidade para as características peso ao nascimento (PN), e ganho de peso do nascimento a desmama (GPND)

Table 1 - Variance estimates and heritabilities for the birth weight $(B W)$ and weight gain from birth to weaning (WGBW)

\begin{tabular}{lcc}
\hline Dados & PN & GPND \\
Data & $B W$ & $W G B W$ \\
\hline $\begin{array}{l}\text { Variância direta } \\
\begin{array}{l}\text { Direct variance } \\
\text { Variância materna }\end{array}\end{array}$ & 5,62 & 85,44 \\
$\begin{array}{l}\text { Maternal variance } \\
\text { Covariância direto-materno }\end{array}$ & 1,26 & 37,03 \\
$\begin{array}{l}\text { Direct-maternal covariance } \\
\begin{array}{l}\text { Herdabilidade direta } \\
\text { Directheritability }\end{array}\end{array}$ & 0,54 & $-2,69$ \\
$\begin{array}{l}\text { Herdabilidade materna } \\
\begin{array}{l}\text { Maternal heritability } \\
\text { Correlação direto-materna } \\
\text { Direct-maternal correlation }\end{array}\end{array}$ & 0,12 & 0,12 \\
\hline
\end{tabular}

R. Bras. Zootec., v.31, n.1, p.321-330, 2002 (suplemento) literatura, demonstrando pequena influência da genética da vaca sobre o desempenho dos indivíduos no peso ao nascer. Esse resultado era esperado, pois, normalmente, quando existe uma alta influência da $\mathrm{h}^{2}$ direta, a materna fica em valores mais baixos. Foi observada também correlação negativa e alta, evidenciando que a $\mathrm{h}^{2}$ materna se torna expressiva somente quando a direta não é influente. Também, para as demais características estudadas, foram evidenciadas correlações negativas.

Coeficientes de herdabilidades(h2) diretas mais baixas, variando de 0,14 a 0,36 e maternas semelhantes foram estimadas para a característica peso ao nascer por Euclides Filho et al. (1978), Eler et al. (1995), e Scarpati \& Lôbo (1999) para a raça Nelore, Marques et al. (1999), para a raça Simental; Ferreira et al. (1999), para a raça Hereford, e Mascioli et al. (1996) para a raça Canchim. Apenas Mascioli et al. (1997) encontraram valor mais alto para o coeficiente de $\mathrm{h}^{2}$ direta para peso ao nascer em um rebanho Canchim, da ordem de 0,76.

Para a característica ganho de peso do nascimento à desmama, pode-se observar (Tabela 1) que tanto a $h^{2}$ direta $(0,12)$ quanto a $h^{2}$ materna $(0,05)$ foram bastante baixas, o que sugere grande influência do meio ambiente nesta fase, indicando que pode ser obtida melhor resposta dos animais desta população quando utilizados outros métodos de melhoramento que não a seleção, como o cruzamento com animais de outros rebanhos Charolês, sendo assim introduzidos novos genes desta raça na população. Valor mais alto, 0,37, foi encontrado por Mascioli et al. (1997), trabalhando com animais Canchim.

Existem poucos trabalhos disponíveis que utilizaram o ganho de peso do nascimento à desmama, porém, características de crescimento à desmama pouco diferem quanto aos valores de coeficientes de herdabilidade, sendo na sua grande maioria, baixos ou de médio a baixo. Para a característica peso à desmama, por exemplo, Dodenhoff et al. (1999) encontraram $h^{2}$ direta de 0,11 e materna de 0,14 para animais da raça Charolês; Keeton et al. (1996) encontraram $\mathrm{h}^{2}$ direta de $0,25 \mathrm{e} \mathrm{h}^{2}$ materna de 0,19 para Limousin; Eler et al. (1995) encontraram $\mathrm{h}^{2}$ direta e materna de 0,13 para animais Nelore; Euclides Filho et al. (1978) encontraram $\mathrm{h}^{2}$ direta de 0,19; e Mercadante \& Lôbo (1997) encontraram $\mathrm{h}^{2}$ direta de 0,29 e materna de 0,13 ambos também para animais Nelore.

De modo geral, os coeficientes de herdabilidade direta e materna foram baixas, com exceção da 
direta para o peso ao nascimento, o que, segundo Oliveira et al. (1995), sugere que a influência da variância genética aditiva na fenotípica total de tais características nesta população é pequena, de modo que o valor fenotípico do indivíduo não deve ser, isoladamente, um indicador de seu valor genético.

As DEP's (diferença esperada na progênie) diretas e maternas para as características estudadas são mostradas na Tabela 2 .

Para a característica peso ao nascimento, a DEP direta estimada foi $-0,0249 \mathrm{~kg}$, com uma variância de $2,3 \mathrm{~kg}$, o que mostra haver pouca variação para esta característica. A DEP materna foi de $0,0092 \mathrm{~kg}$, o que indica baixa influência dos genes da mãe sobre o desempenho dos animais desta população para esta característica.

Para a característica ganho de peso do nascimento à desmama, a DEP direta média foi de $-0,1637$; valor este bastante próximo de zero, a exemplo das DEP's médias, direta e materna, de todas as características estudadas. A DEP materna apresentou menor variação, devido, provavelmente, à pequena influência da genética da vaca sobre esta característica de crescimento.

Torna-se interessante ressaltar a distribuição bastante uniforme dos animais acima e abaixo da média, para o GPND, com os valores de DEP's maternas máxima e mínimas da ordem de 3,44 e -3,50 respectivamente, com a média próxima de zero.

Tabela 2 - Estimativas de DEP's (diferença esperada na progênie) para as características peso ao nascimento (PN), e ganho de peso do nascimento a desmama (GPND)

Table 2 - EPD (expected predict difference) estimates for the birth weight $(B W)$ and weight gain from birth to weaning (WGBW)

\begin{tabular}{|c|c|c|c|c|}
\hline \multirow{3}{*}{$\begin{array}{l}\text { Direta } \\
\text { Direct }\end{array}$} & \multicolumn{2}{|c|}{$\mathrm{PN}(B W)$} & \multicolumn{2}{|c|}{ GPND $(W G B W)$} \\
\hline & Materna & Direta & Materna & Direta \\
\hline & Maternal & Direct & Maternal & Direct \\
\hline $\mathrm{N}^{\mathrm{o}}$ de observações & 6529 & 6529 & 9808 & 9808 \\
\hline N. of observations & & & & \\
\hline DEP Média & $-0,0249$ & 0,0092 & $-0,1637$ & 0,0344 \\
\hline $\begin{array}{l}\text { Mean EPD } \\
\text { EFP mínima }\end{array}$ & & & & \\
\hline $\begin{array}{l}\text { DEP mínima } \\
\text { Minimum EPD }\end{array}$ & $-10,493$ & $-1,703$ & $-13,718$ & $-3,502$ \\
\hline $\begin{array}{l}\text { DEP máxima } \\
\text { Maximum EPD }\end{array}$ & 8,428 & 2,297 & 15,175 & 3,443 \\
\hline $\begin{array}{l}\text { Variância } \\
\text { Variance }\end{array}$ & 2,3042 & 0,1128 & 17,5750 & 0,6451 \\
\hline
\end{tabular}

R. Bras. Zootec., v.31, n.1, p.321-330, 2002 (suplemento)
DEP's médias próximas de zero também foram encontradas por Núñez-Dominguez et al. (1993), para peso ao nascer, trabalhando com várias raças, e peso à desmama, para a raça Charolês; e por Barkhouse et al. (1998), também para a raça Charolês, para a característica peso ao nascer, porém estes mesmos autores encontraram valores distantes de zero, para as DEP's médias para o peso à desmama.

Ao analisar as DEP's diretas individuais das características peso ao nascer e ganho de peso do nascimento à desmama, foi verificada correlação positiva e altamente significativa $(\mathrm{P}<0,01)$ de 0,12881 , indicando que, quanto maiores as DEP's para o peso ao nascer, maiores também serão as DEP's para o ganho de peso.

Uma DEP positiva para a característica ganho de peso é bastante almejada, porém o ideal para esta raça, devido à elevada taxa de distocia causada pelo grande tamanho dos terneiros ao nascer, é que, para o peso ao nascer, a DEP seja negativa, logo esta correlação não se torna interessante. Entretanto, foi verificada, após ordenação dos animais de acordo com as suas DEP's diretas, a presença de indivíduos com alto valor seletivo, se adotado o critério de seleção do baixo peso ao nascer e do alto ganho de peso do nascer à desmama.

De acordo com os dados mostrados na Tabela 3, existem animais nesta população, como o animal 08 , que conseguiriam satisfazer tanto a exigência de menor peso ao nascer como a de não diminuição dos pesos subseqüentes, na próxima geração, já que a expectativa seria de que os seus descendentes apresentassem, em média, $5,7 \mathrm{~kg}$ a menos no peso ao nascer que a média do rebanho e 7,3 $\mathrm{kg}$ a mais que a média do rebanho para a característica ganho do nascimento à desmama, isto é, estes animais compensariam o menor peso ao nascer até a época da desmama.

As tendências genéticas diretas observadas para esta população foram de $-10,46 \mathrm{~g}$ anuais para o peso ao nascimento (Figura 3), e de 40,85 g anuais (Figura 4) para a característica ganho de peso do nascimento à desmama, o que representa 0,025 e $0,026 \%$ em relação às médias do peso ao nascer e do ganho de peso pré-desmama, respectivamente.

Estas tendências não foram significativas $(\mathrm{P}>0,10)$, o que indica que estes progressos anuais, positivos ou negativos, foram muitos pequenos, ou seja, os resultados permitem que se defina este rebanho como estagnado quanto à fração genética, para as características estudadas, não estando 
Tabela 3 - Valores de DEP (diferença esperada na progênie) para as características peso ao nascimento (PN) e ganho de peso do nascimento à desmama (GPND) de alguns animais da população estudada

Table 3 - EPD (expected predict difference) values for the characteristics birth weight (BW), and weight gain from birth to weaning (WGBW) of few animals of the studied population

\begin{tabular}{lcc}
\hline Animal & DEP-PN & DEP-GPND \\
Animal & $E P D-B W$ & $E P-W G B W$ \\
\hline 01 & $-8,788$ & 2,788 \\
02 & $-7,513$ & 1,758 \\
03 & $-7,183$ & 4,080 \\
04 & $-6,826$ & 2,576 \\
05 & $-6,636$ & 5,775 \\
06 & $-6,253$ & 4,278 \\
07 & $-5,964$ & 3,198 \\
08 & $-5,754$ & 7,331 \\
09 & $-5,230$ & 4,251 \\
10 & $-5,174$ & 4,593 \\
11 & $-5,112$ & 5,832 \\
12 & $-4,522$ & 4,571 \\
13 & $-4,386$ & 6,224 \\
14 & $-4,330$ & 3,766 \\
15 & $-4,076$ & 4,749 \\
16 & $-4,021$ & 4,497 \\
17 & $-3,553$ & 4,991 \\
18 & $-3,536$ & 6,454 \\
19 & $-3,520$ & 8,621 \\
20 & $-3,385$ & 10,903 \\
21 & $-3,259$ & 5,075 \\
22 & $-3,238$ & 5,170 \\
23 & $-3,154$ & 5,611 \\
24 & $-3,086$ & 8,182 \\
25 & $-2,560$ & 6,648 \\
\hline & &
\end{tabular}

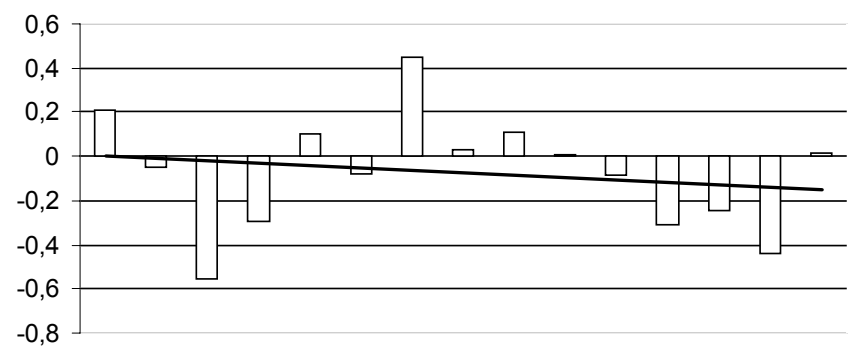

Eixo vertical: valores de DEP's anuais médias; eixo horizontal: ano (de 1984 a 1998); equação de regressão: $-0,01046 x+0,01582 ;$ significância de 0,5201 .

Vertical axis: values of means annuals for EPD's; horizontal axis: year (from 1984 to 1998); regression equation: $.01046 x+.01582$; significance of .5201 .

Figura 3 - DEP's diretas médias anuais (1984 a 1998) com a respectiva reta de tendência genética direta para o peso ao nascimento.

Figure 3 - Direct means annual EPD's (1984 to 1998) and the respective tendency genetic line for the birth weigh.

R. Bras. Zootec., v.31, n.1, p.321-330, 2002 (suplemento) regredindo, porém também não estando progredindo.

A tendência genética materna para o peso ao nascimento (2,38 g/ano) não foi significativa(Figura 5), indicando uma estagnação também da genética das matrizes para esta característica. Entretanto, a tendência genética materna da característica ganho de peso do nascimento à desmama (Figura 6) foi significativa $(\mathrm{P}<0,001)$ de $-10,34 \mathrm{~g} / \mathrm{ano}$, indicando que a genética das vacas desta população vem regredindo com o passar dos anos e irá continuar decaindo futuramente, se não for adotado um programa de melhoramento genético para esta característica.

Para a característica peso ao nascimento, Oliveira et al. (1995) encontraram tendência direta de $13 \mathrm{~g} /$ ano para animais da raça Guzerá, o que correspondeu a $0,05 \%$ da média desta característica; Ledic et al. (1988) verificaram tendência direta de 23,6 g/ano para um rebanho Tabapuã; Scarpati et al. (1998) observaram tendência direta de $40 \mathrm{~g}$ /ano e materna de $5 \mathrm{~g}$ /ano para animais Nelore; Sullivan et al. (1999), estimaram tendência direta de $130 \mathrm{~g}$ /ano para Charolês e $40 \mathrm{~g}$ /ano para Limousin; Mello (1999) verificaram tendência direta de $46 \mathrm{~g} / \mathrm{ano}$ e materna de $-2 \mathrm{~g} / \mathrm{ano}$ para animais Canchim $(\mathrm{P}<0,05)$; Euclides Filho et al. (1986) observaram tendência direta de -44 g/ano para um rebanho Nelore.

Trabalhando com a característica ganho de peso do nascimento à desmama, Euclides Filho et al. (1997a)

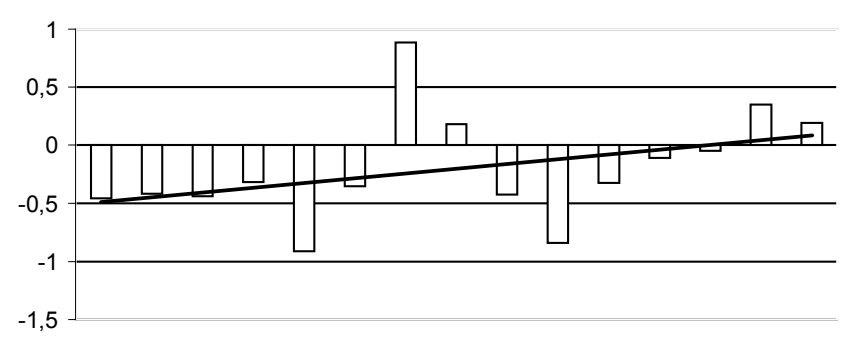

Eixo vertical:valores de DEP's anuais médias; eixo horizontal: ano (de 1984 a 1998); equação de regressão: $0,04085 x+0,02641$; significância de 0,1459 .

Vertical axis: values of means annuals for EPD's; horizontal axis: year (from 1984 to 1998); regression equation: .04085x +.02641 ; significance of .1459 .

Figura 4 - DEP's diretas médias anuais (1984 a 1998) com reta de tendência genética direta para o ganho de peso do nascimento à desmama.

Figure 4 - Direct means annual EPD's (1984 to 1998) and the respective tendency genetic line for the weight gain from birth to weaning. 


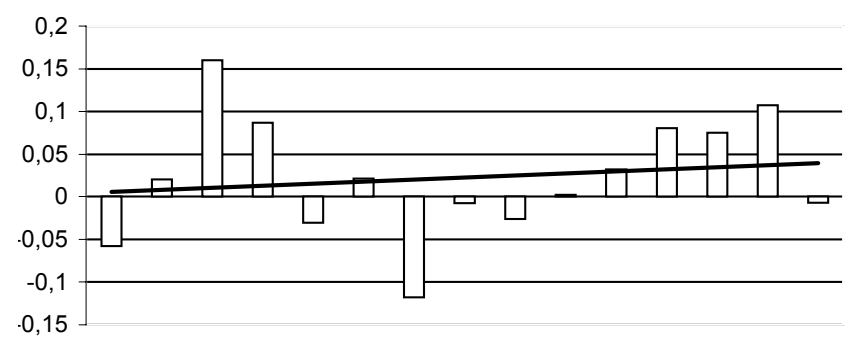

Eixo vertical: valores de DEP's anuais médias; eixo horizontal: ano (de 1984 a 1998); equação de regressão: 0,00238x + 0,00433; significância de 0,5924 .

Vertical axis: values of means annuals for EPD's; horizontal axis: year (from 1984 to 1998); regression equation: .00238x +.00433 ; significance of .5924 .

Figura 5 - DEP's maternas médias anuais (1984 a 1998) com a respectiva reta de tendência genética materna para o peso ao nascimento.

Figure 5 - Maternal means annual EPD's (1984 to 1998) and the respective tendency genetic line for birth weight.

encontraram tendência direta de $67 \mathrm{~g} /$ ano $(\mathrm{P}<0,05)$ e tendência materna de $36,5 \mathrm{~g} /$ ano $(\mathrm{P}<0,05)$ para animais da raça Guzerá; Euclides Filho et al. (2000) estimaram tendência direta de $68,84 \mathrm{~g} / \mathrm{ano}(\mathrm{P}<0,05)$, o que representa $0,04 \%$ da média do ganho de peso para esta população de animais da raça Gir e tendência

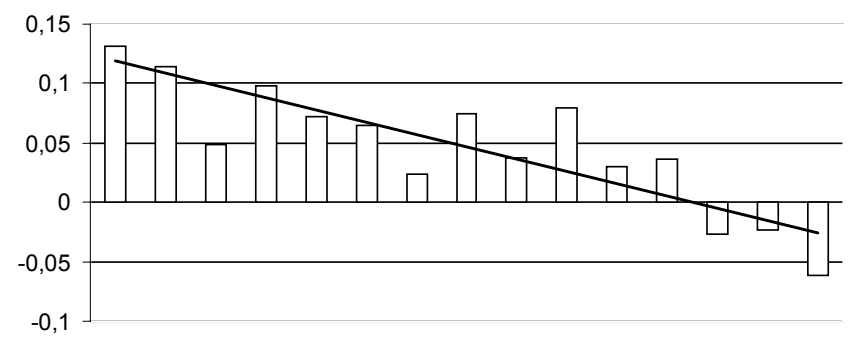

Eixo vertical: valores de DEP's anuais médias; eixo horizontal: ano (de 1984 a 1998); equação de regressão: $-0,01034 x+0,00169$; significância de 0,0001.

Vertical axis: values of means annuals for EPD's; horizontal axis: year (from 1984 to 1998); regression equation: $-.01034 x+.00169$; significance of .0001 .

Figura 6 - DEP's maternas médias anuais (1984 a 1998) com reta de tendência materna para o ganho de peso do nascimento a desmama.

Figure 6 - Maternal means annual EPD's (1984 to 1998) and the respective tendency genetic line for weight gain from birth to weaning.

R. Bras. Zootec., v.31, n.1, p.321-330, 2002 (suplemento)

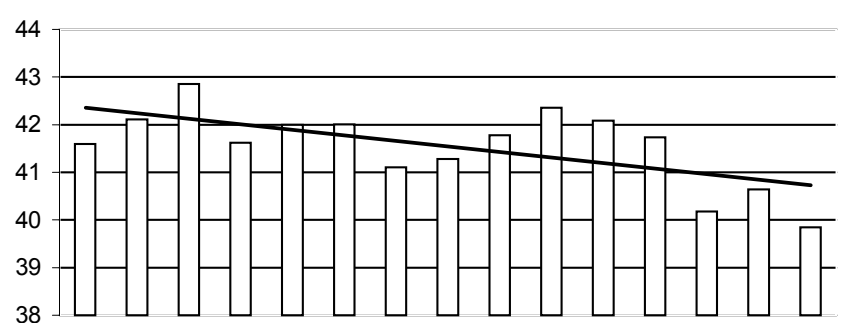

Eixo vertical: valores de DEP's anuais médias; eixo horizontal: ano (de 1984 a 1998); equação de regressão: $-0,11560 x+0,03917$; significância de 0,0113 .

Vertical axis: values of means annuals for EPD's; horizontal axis: year (from 1984 to 1998); regression equation: $.011560 x+.03917$; significance of $.0,0113$.

Figura 7 -Médias totais anuais (1984 a 1998) com a respectiva reta de tendência total para o peso ao nascimento.

Figure 7 - Total means annual EPD's (1984 to 1998) and the respective tendency genetic line for birth weight.

materna de $21,75 \mathrm{~g}$ /ano, equivalente a $0,01 \%$ da média; Silva et al. (1997b) encontraram tendência direta de $259 \mathrm{~g}$ /ano $(\mathrm{P}<0,05)$ e materna de $226 \mathrm{~g} /$ ano $(\mathrm{P}<0,05)$ trabalhando com animais Nelore; e Euclides Filho et al. (1997b) estimaram tendência direta de $90 \mathrm{~g} / \mathrm{ano}$, o que representa $0,06 \%$ da média, e materna de $24 \mathrm{~g} /$ ano $(0,01 \%$ da média), para animais Indubrasil.

De modo geral, os valores apresentaram-se bem abaixo do potencial, que é, segundo Smith (1985), dentro de um intervalo de 1 a $3 \%$ da média da população para a taxa anual de mudança genética.

A tendência total ou fenotípica, ou seja, a porção genética e não genética da população, considerando a influência do meio ambiente, foi de $-0,12 \mathrm{~kg}(\mathrm{P}<0,02)$ para a característica peso ao nascer (Figura 7), e de $3,90 \mathrm{~kg}(\mathrm{P}<0,0001)$ para a característica ganho de peso do nascimento à desmama (Figura 8). Nota-se que as características estão melhorando com o tempo, ou seja, o peso ao nascer está diminuindo e o ganho de peso está aumentando. Entretanto, embora os produtores estejam conseguindo progresso anual significativo no desempenho dos seus animais, analisados neste estudo, este resultado é devido quase que totalmente à melhoria nas condições de meio ambiente a que estes estão sendo submetidos, uma vez que a porção genética, como já foi colocado, não está contribuindo para que ocorra esta evolução, logo, esta evolução tende a atingir o seu limite, se não houver melhoria genética nos próximos anos. 


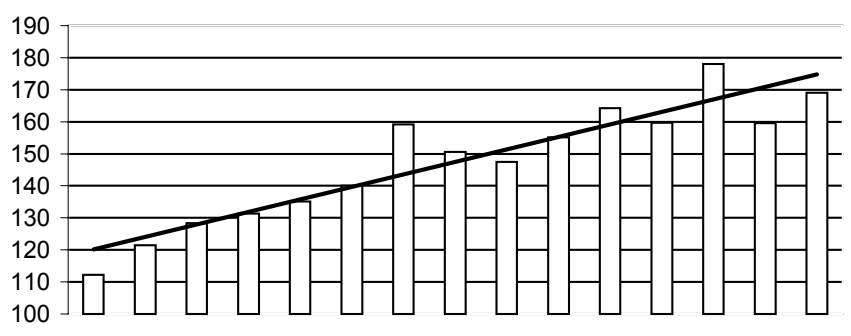

Eixo vertical: valores de DEP's anuais médias; eixo horizontal: ano (de 1984 a 1998); equação de regressão: 3,90032x + 0,42468; significância de 0,0001 .

Vertical axis: values of means annuals for EPD's; horizontal axis: year (from 1984 to 1998); regression equation: $3.90032 x+.42468$; significance of .0001 .

Figura 8 - Médias totais anuais (1984 a 1998) com respectiva reta de tendência total para o ganho de peso do nascimento a desmama

Figure 8 - Total means annual EPD's (1984 to 1998) and the respective tendency genetic line for weight gain from birth to weaning.

\section{Conclusões}

Devido à não significância das alterações anuais verificadas na genética dos animais, pode-se inferir que o rebanho se encontra estagnado em relação às características peso ao nascer e ganho de peso do nascimento à desmama.

Existem animais melhoradores na população que podem ser selecionados, levando-se em consideração o critério de baixa DEP para peso ao nascer e alta para o ganho de peso do nascimento à desmama.

É de suma importância a rapidez na adoção de um programa de melhoramento genético, devido ao fato de a genética materna para o ganho de peso do nascimento à desmama estar regredindo significativamente.

Devido ao fato de estar ocorrendo progresso fenotípico significativo para estas características, evidenciando uma preocupação por parte dos produtores com a melhoria do meio ambiente proporcionado aos animais. Torna-se, por conseguinte, mais recomendável a adoção de um programa de melhoramento genético, pois seria mais fácil a obtenção de bons resultados.

Os resultados indicam que mesmo rebanhos com alto progresso fenotípico podem apresentar pouco progresso genético, o que salienta a importância da realização de outros trabalhos seguindo esta linha de pesquisa, com maior número de animais e também utilizando outras raças, para que o rebanho bovino gaúcho tenha maior acompanhamento, verificando assim se está ocorrendo progresso genético e fenotípico próximo ao que pode ser alcançado;

É recomendável que se dê igual importância à melhoria genética do rebanho e do meio ambiente a que os animais são submetidos, para que se consiga progresso contínuo da população no decorrer dos anos.

\section{Literatura Citada}

BARKHOUSE, K.L.; Van VLECK, L.D.; CUNDIFF, L.V. et al. Comparison of breed solutions for growth traits adjusted by mean expected progeny differences to a 1993 base. Journal of Animal Science, v.76, p.2287-2293, 1998.

BIFFANI, S.; MARTINS FILHO, R.; GIORGETTI, A. et al. Fatores ambientais e genéticos sobre o crescimento ao ano e ao sobreano de bovinos Nelore, criados no nordeste do Brasil. Revista Brasileira de Zootecnia, v.28, n.3, p.468-473, 1999.

BOLDMAN, K.G.; KRIESE, L.A.; Van VLECK, L.D. et al. A manual for use of MTDFREML: a set of programs to obtain estimates of variance and covariance. Lincoln: Department of Agriculture/ Research Service, 1995. 120p.

DODENHOFF, J.; Van VLECK, L.D.; GREGORY, K.E. Estimation of direct, maternal, and grandmaternal genetic effects for weaning weight in several breeds of beef cattle. Journal of Animal Science, v.77, p.840-845, 1999.

ELER, J.P.; Van VLECK, L.D.; FERRAZ, J.B.S. et al. Estimation of variance due to direct and maternal effects for growth traits of Nelore cattle. Journal of Animal Science, v.73, p.32533258, 1995.

EUCLIDES FILHO, K.; SILVA, M.A.; MILAGRES, J.C. Estimativas de parâmetros genéticos e fenotípicos de pesos e ganhos de peso durante o aleitamento. Revista da Sociedade Brasileira de Zootecnia, v.7, n.2, p.234-244, 1978.

EUCLIDES FILHO, K.; NOBRE, P.R.C.; ROSA, A.N. Tendências genéticas em características de crescimento de gado Nelore. In: REUNIÃO ANUAL DA SOCIEDADE BRASILEIRA DE ZOOTECNIA, 23., 1986, Campo Grande. Anais... Campo Grande: Sociedade Brasileira de Zootecnia, 1986. p.311.

EUCLIDES FILHO, K.; SILVA, L.O.C.; FIGUEIREDO, G.R. Tendências genéticas na raça Guzerá. In: REUNIÃO ANUAL DA SOCIEDADE BRASILEIRA DE ZOOTECNIA, 34., 1997, Juiz de Fora. Anais... Juiz de Fora: Sociedade Brasileira de Zootecnia 1997a. p.173-174.

EUCLIDES FILHO, K.; SILVA, L.O.C.; ALVES, R.G.O. et al. Tendências genéticas na raça Indubrasil. In: REUNIÃO ANUAL DA SOCIEDADE BRASILEIRA DE ZOOTECNIA, 34., 1997, Juiz de Fora. Anais... Juiz de Fora: Sociedade Brasileira de Zootecnia, 1997b. p.171-172.

EUCLIDES FILHO, K.; SILVA, L.O.C.; ALVES, R.G.O. et al. Tendência genética na raça Gir. Pesquisa Agropecuária Brasileira, v.35, n.4, p.787-791, 2000.

FERNANDES, H.D.; FERREIRA, G.B. Estudo comparativo de sete diferentes modelos estatísticos para a característica ganho de peso em bovinos de corte. Revista Brasileira de Zootecnia, v.29, n.5, p.1340-1348, 2000.

FERREIRA, G.B.; MacNEIL, M.D.; Van VLECK, L.D. Variance components and breeding values for growth traits from different statistical models. Journal of Animal Science, v.77, p.2641-2650, 1999.

FRIES, L.A.; ALBUQUERQUE, L. Pressuposições e restrições 
dos modelos animais com efeito materno em gado de corte. In: WORKSHOP COMPORTAMENTO MATERNO EM MAMÍfEROS, 1996, Jaboticabal. Proceedings... Jaboticabal: UNESP, 1996.

KEETON, L.L.; GREEN, R.D.; GOLDEN, B.L. et al. Estimation of variance components and prediction of breeding values for scrotal circumference and weaning weight in Limousin cattle. Journal of Animal Science, v.74, p.31-36, 1996.

LEDIC, I.L.; NOBRE, P.R.C.; ROSA, A.N. Tendências fenotípicas, ambientes e genéticas estimadas para os pesos ao nascer (PN), aos 205 (P205), 365 (P365) e 550 (P550) dias de idade de animais da raça Tabapuã. In: REUNIÃO ANUAL DA SOCIEDADE BRASILEIRA DE ZOOTECNIA, 25., 1988, Viçosa. Anais... Viçosa, MG: Sociedade Brasileira de Zootecnia, 1988. p.238.

MARQUES, L.F.A.; PEREIRA, J.C.C.; OLIVEIRA, H.N. et al. Componentes de (co)Variância e parâmetros genéticos de características de crescimento da raça Simental na Brasil. Arquivo Brasileiro de Medicina Veterinária e Zootecnia, v.51, n.4, p.363-370, 1999.

MASCIOLI, A.S.; ALENCAR, M.M.; BARBOSA, P.F. et al. Estimativas de parâmetros genéticos e proposição de critério de seleção para pesos na raça Canchim. Revista da Sociedade Brasileira de Zootecnia, v.25, n.1, p.72-82, 1996.

MASCIOLI, A.S.; PAZ, C.C.P.; EL FARO, L. et al. Estimativas de parâmetros genéticos e fenotípicos para características de crescimento até a desmama em bovinos da raça Canchim. Revista Brasileira de Zootecnia, v.26, n.4, p.709-713, 1997.

MELLO, S.P. Tendência genética para pesos em um rebanho da raça Canchim. Jaboticabal: Universidade Estadual Paulista, 1999. 78p. Dissertação (Mestrado em Zootecnia) - Universidade Estadual Paulista, 1999.

MERCADANTE, M.E.Z.; LÔBO, R.B. Estimativas de (co)Variância e parâmetros genéticos dos efeitos direto e materno de características de crescimento de fêmeas de um rebanho Nelore. Revista Brasileira de Zootecnia, v.26, n.6, p.1124-1133, 1997.

MOTA, F.S.; ZAHLER, P.J.M. Clima, agricultura e pecuária no Rio Grande do Sul. Pelotas: 1994. 166p.

NÚÑEZ-DOMINGUEZ, R.; Van VLECK, L.D.; CUNDIFF, L.V. Breed comparison for growth traits adjusted for withinbreed genetic trend using expected progeny differences. Journal of Animal Science, v.71, p.1419-1428, 1993.
OLIVEIRA, J.A.; LÔBO, R.B.; OLIVEIRA, H.N. Tendência genética em pesos e ganhos em peso de bovinos da raça Guzerá. Pesquisa Agropecuária Brasileira, v.30, n.11, p.1355-1360, 1995.

SAS INSTITUTE. Statistical analysis systems user's guide: Statistical, Version 6.11 ed. Cary: 1996.

SCARPATI, M.T.V.; LÔBO, R.B.; OLIVEIRA, H.N. et al. Tendência genética do período de gestação e peso ao nascer em rebanhos da raça Nelore. In: SIMPÓSIO NACIONAL DA SOCIEDADE BRASILEIRA DE MELHORAMENTO ANIMAL, 2., 1998, Uberaba. Anais... Uberaba: Sociedade Brasileira de Melhoramento Animal, 1998. p.381-382.

SCARPATI, M.T.V.; LÔBO, R.B. Modelos animais alternativos para estimação de componentes de (co)Variância e de parâmetros genéticos e fenotípicos do peso ao nascer na raça Nelore. Revista Brasileira de Zootecnia, v.28, n.3, p.512-518, 1999.

SILVA, L.O.C.; EUCLIDES FILHO, K.; FIGUEIREDO, G.R. et al. Tendências genéticas na raça Tabapuã no Brasil. In: REUNIÃO ANUAL DA SOCIEDADE BRASILEIRA DE ZOOTECNIA, 34., 1997, Juiz de Fora. Anais... Juiz de Fora: Sociedade Brasileira de Zootecnia, 1997a. p.177-179.

SILVA, L.O.C.; EUCLIDES FILHO, K.; NOBRE, P.R.C. et al. Tendências genéticas na raça Nelore no Brasil. In: REUNIÃO ANUAL DA SOCIEDADE BRASILEIRA DE ZOOTECNIA, 34., 1997, Juiz de Fora. Anais... Juiz de Fora: Sociedade Brasileira de Zootecnia, 1997b. p.175-176.

SMITH, C. Rates of genetic changes in farm livestock. Research and Development Agriculture, v.1, n.2, p.79-85, 1985.

SULLIVAN, P.G.; WILTON, J.W.; MILLER, S.P. et al. Genetic trends and breed overlap derived from evaluations of breed cattle for multiple-breed genetic growth traits. Journal of Animal Science, v.77, n.8, p.2019-2027, 1999.
Recebido em: 29/03/01 Aceito em: 17/10/01 